\title{
Multi-objective optimization and linear buckling of serial chain of a medical robot tool for soft tissue surgery
}

\author{
Grzegorz Ilewicz, Andrzej Harlecki \\ Division of Mechanics, Faculty of Mechanical Engineering and Computer Sciences, University of Bielsko-Biala, Poland
}

\begin{tabular}{l}
\hline Article Info \\
\hline Article history: \\
Received Oct 13, 2019 \\
Revised Dec 27, 2019 \\
Accepted Jan 12, 2020 \\
\hline
\end{tabular}

\section{Keywords:}

Eigenvalue problem

Finite element method

Linear buckling

Medical robot

MOGA genetic algorithm

Optimization

Pareto front

\begin{abstract}
The slender structures of a medical robot may have a tendency to buckling when a force equal to the critical Euler force and an additional disturbance will work on their structures. In this work, eigenvalue problem that describes the linear buckling is under consideration. The main goal of the article is to check when linear buckling phenomenon appears in construction of a medical robot with serial chain due to the fact that for safety reasons of a robot's work, it is necessary to answer the question, whether the buckling may occur in the robot's structure. For this purpose, a numerical calculation model was defined by using the finite element method. The values of load factor coefficients that are eigenvalue are determinated and also the eigenvectors that have shapes of deformation for the next eigenvalues are presented. The multi-criteria optimization model was determined to aim for the minimum mass of the effector and the buckling coefficient, from which the Euler force results, for the maximum. The solution was obtained on the basis of Pareto fronts and the MOGA genetic algorithm.
\end{abstract}

This is an open access article under the CC BY-SA license.

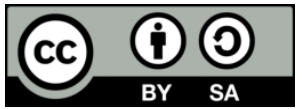

\section{Corresponding Author:}

Grzegorz Ilewicz,

Department of Mechanics,

Faculty of Mechanical Engineering and Computer Sciences,

Ul.Willowa 2, Bielsko-Biala, Poland.

Email: gilewicz@ath.bielsko.pl

\section{INTRODUCTION}

Constructions of medical robots are being increasingly considered scientifically for operations of the human body and for servicing the artificial organs having an open kinematic chain. In this way, there is a progressive search to those currently existing in clinical practice. This paper aims to answer the question of whether the buckling can appear in the construction of a medial robot with an open kinematic chain. The examination of buckling is a very important experience in the construction of a robot due to the fact that the safety criterion of the usage of a medical robot during surgery is very restrictive. Having no answer to this question about buckling, makes it impossible to use a medical robot for surgeries on the human body due to the safety of a patient and the operating team which is an overriding validity.

This mini robot is attached to the main mechanism of the maxi robot that is called a constant point mechanism. The constant point mechanism is shown in the Figure 1(a). This mechanism has three degrees of freedom for regional movement. The mini robot with six degrees of freedom is to manipulate the soft tissue. The main working space, which results from the first three degrees of freedom, has a shape which is shown in Figure 1(b). Inside this volume, the kinematic chain of a mini robot with the RRRS configuration is moving.

The mathematical formula that describes working space is described as: 


$$
|V|=4 \int_{0}^{\frac{\varphi_{1}}{2}} d \varphi_{1} \int_{\varphi_{2}}^{\frac{\pi}{2}} \sin \left(\varphi_{2}\right) d \varphi_{2} \int_{0}^{\lambda} \lambda^{2} d \lambda
$$

where: $\varphi_{1}, \varphi_{2}, \lambda$ - joints variables in the next degrees of freedom. From the volume of the working space results the length of the mini robot's arm, shown in Figure 2. Due to the slim shape after the stretch and thin walls of the subsequent links of the robot, it may be suspected that the buckling may appear in the structure, e.g. during the servicing of an artificial organ.

Model of a vector optimization using MOGA genetic algorithm, where three criteria were important: the first natural frequency in order to increase stiffness of the tool, mass that may minimize inertial forces and equivalent stress in transient states was described in work [1, 2]. Important information about elastic buckling problems is announced in work [3-5]. The usage of Pareto fronts for the optimization of a medical robot is illustrated in work [6-9]. There is also a work, where the Lanchos method is used for solving eigenvalue problems and is described in [10]. The mathematical basis of the finite element method is explained in work [11]. The finite element method is used for calculating the elastic buckling problem because it gives the correct solution and the time of computations is not so long when the geometrical model of the object is actually simplified.

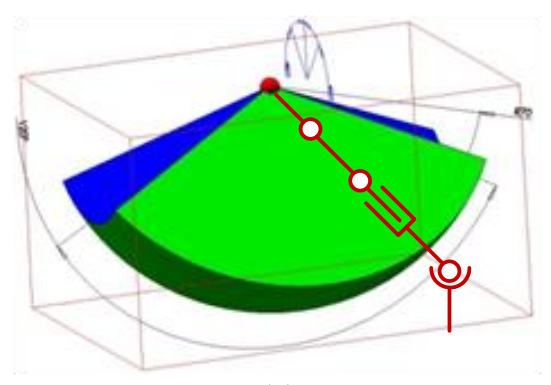

(a)

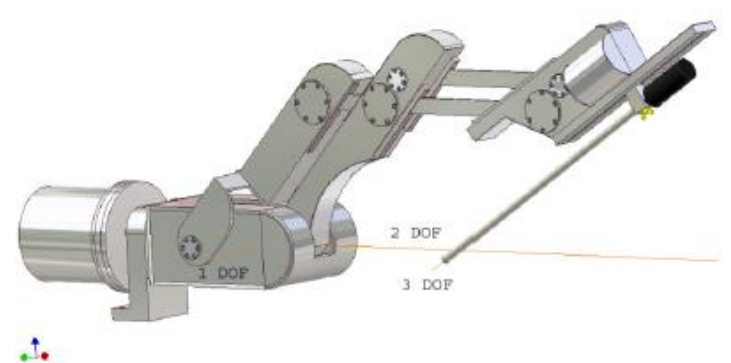

(b)

Figure 1. (a) Constant point mechanism and (b) shape of the working space

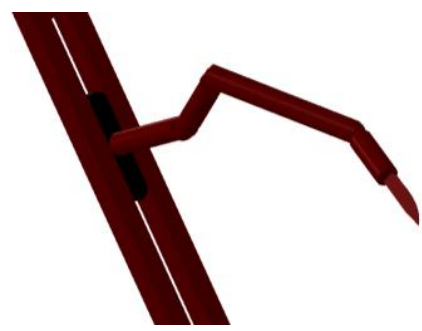

Figure 2. Model of mini medical robot with serial chain

\section{RESEARCH METHOD}

The differential equation describing the own buckling problem is formulated as:

$$
E J \frac{d^{2} y}{d z^{2}}+F y=0
$$

where:

E- Young's modulus,

$\mathrm{J}$ - moment of inertia,

$F$ - external force.

By converting (2) and substituting (3) to (2):

$$
\frac{F}{E J}=k^{2}
$$


an equation is obtained:

$$
\frac{d^{2} y}{d z^{2}}+k^{2} y=0
$$

The general solution of (4) has a trigonometric form:

$$
y=A \cos (k z)+B \sin (k z)
$$

If we take (3), and we know that:

$$
k l=\pi, 2 \pi, \ldots, n \pi
$$

where $n$ is a natural number, we can obtain Euler's critical force:

$$
F_{c r}=k^{2} E J=\frac{\pi^{2} E J}{l^{2}}
$$

The force that is given by (7) is the force which causes elastic buckling in thin rod of length 1 , when to the rod will be applied any disturbance. If to a thin rod is applied any force, it is important to define lambda coefficient of load. The value of critical Euler's load can be determined as:

$$
F_{c r}=\lambda \cdot F
$$

The load coefficient $\lambda$ is written as:

$$
\lambda=\frac{F_{k r}}{F}
$$

The linear buckling, which scheme is shown by Figure 3, is determined by following conditions:

$$
\lambda<1 \text { - conditions of buckling (instability) }
$$

$$
\lambda>1 \text { - conditions of stability }
$$

If (10) occurs and any disturbance is added, a buckling will appear. If (11) occurs, the medical robot will be a stable mechanical system.

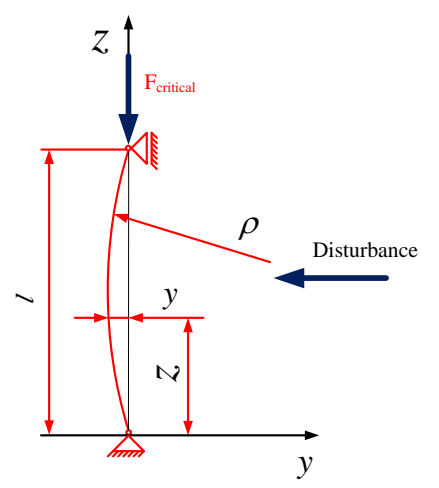

Figure 3. Scheme of linear buckling

\subsection{Eigenvalue problem of linear buckling using the finite element method}

The buckling coefficient $\lambda$ can be calculated using the finite element method but taking into the account the equation of static equilibrium, having in mind small displacements. The relationship between external force and displacement can be recorded as:

$$
[\boldsymbol{K}] \cdot\{\boldsymbol{u}\}=\{\boldsymbol{F}\}
$$

where: 
$[\boldsymbol{K}]$ : stiffness matrix,

$\{\boldsymbol{u}\}$ : vector of nodal displacement,

$\{\boldsymbol{F}\}$ : vector of nodal forces.

The stiffness matrix $[\boldsymbol{K}]$ is determined by:

$$
[\boldsymbol{K}]=\int_{V}[\boldsymbol{B}]^{T}[\boldsymbol{D}][\boldsymbol{B}] d V
$$

where:

$[\boldsymbol{B}]$ : linear strain - displacement matrix $\{\boldsymbol{\varepsilon}\}=[\mathbf{B}]\{\boldsymbol{d}\}$ (matrix of shape function),

[D] : constitutive matrix,

$\{\boldsymbol{\varepsilon}\}$ : vector of strain,

$\{\boldsymbol{d}\}$ : vector of displacement.

$$
\left[\boldsymbol{K}_{\boldsymbol{G}}\right]=\int_{V}[\boldsymbol{G}]^{T}[\boldsymbol{S}][\boldsymbol{G}] d V^{2}
$$

where:

$\left[\boldsymbol{K}_{\boldsymbol{G}}\right]$ : stress-stiffness matrix,

[G] : obtained from shape functions by appropriate differentiation,

[S] : initial stresses.

$$
\left([\boldsymbol{K}]+\left[\boldsymbol{K}_{\boldsymbol{G}}\right]\right) \cdot\{\boldsymbol{u}\}=\{\boldsymbol{F}\}
$$

During the loss of stability for equal loads, other states of equilibrium are possible.

$$
\begin{aligned}
& \left([\boldsymbol{K}]+\lambda\left[\boldsymbol{K}_{\boldsymbol{G}}\right]\right) \cdot\{\boldsymbol{u}\}=\{\boldsymbol{F}\} \\
& \left([\boldsymbol{K}]+\lambda\left[\boldsymbol{K}_{\boldsymbol{G}}\right]\right) \cdot\{\boldsymbol{u}+\boldsymbol{\delta} \boldsymbol{u}\}=\{\boldsymbol{F}\}
\end{aligned}
$$

After subtracting the equations, the symmetrical problem that defines the stability of the substitution system is obtained to solve:

$$
\left([\boldsymbol{K}]+\lambda\left[\boldsymbol{K}_{G}\right]\right) \cdot\{\boldsymbol{\delta u}\}=\{\boldsymbol{0}\}
$$

$\lambda \quad$ : eigenvalues which are load coefficients,

$\boldsymbol{\delta} \boldsymbol{u}$ : eigenvector which is shape of buckling.

Lanchos method for large symmetrical systems was used in order to solve the problem described above.

\subsection{Model of the tool}

To reduce the time of numerical calculations, the clearing geometry was used and the time of numerical calculations was significantly reduced. The formulated numerical model has 454534 degrees of freedom. Ten nodal tetrahedral elements were used to discretize the model. The model was supported on the plane A (fixed support). In point B, a force of 30 [N] was applied as a load. This force will cause the compression of mechanical system of a mini robot. Figure 4 shows the discrete model of the effector, while Figure 5 shows the boundary conditions of the model.

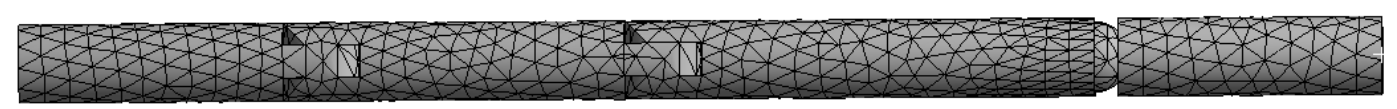

Figure 4. Discrete model of the effector

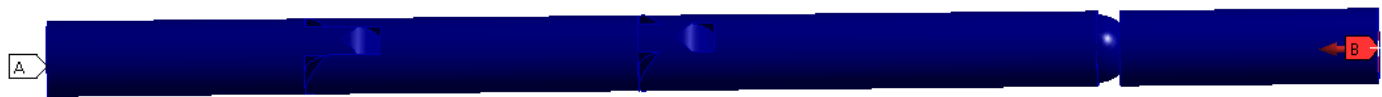

Figure 5. Boundary conditions of the model

Int J Rob \& Autom, Vol. 9, No. 1, March 2020 : 17 - 25 


\subsection{Multi-objective optimization}

The optimization model shown in this work contains two criteria, one of which is the buckling coefficient $\lambda$ and the other is the effector's mass. The aim is to make the buckling coefficient as high as possible while achieving the minimum weight of the mini robot. Such an aspiration will allow to obtain positive static properties of the effector as well as the inertial effects in motion (resulting from the mass) will reach a lower value, which will cause less deformation of the effector, i.e. its greater accuracy of positioning at the tissue.

A multi-criteria objective function was adopted:

$$
\boldsymbol{f}(\{\boldsymbol{d}\})=\left\{\boldsymbol{f}_{\mathbf{1}}\{\boldsymbol{d}\}, \boldsymbol{f}_{\mathbf{2}}\{\boldsymbol{d}\}\right\}
$$

where:

$\boldsymbol{f}_{\mathbf{1}}(\{\boldsymbol{d}\})$ : mass vector,

$\boldsymbol{f}_{2}(\{\boldsymbol{d}\})$ : vector of the first buckling form,

$d_{1}, d_{2}$, : dimensions, from which result the thickness of walls of the first and second.

The restrictions of the model can be specified as:

$4[\mathrm{~mm}] \leq d_{1} \leq 9.9[\mathrm{~mm}]$

$4[\mathrm{~mm}] \leq d_{2} \leq 9.9[\mathrm{~mm}]$.

Also an additional assumption was made that:

$$
\lambda>1
$$

where: $\lambda$ - coefficient of load. The mesh model solutions were made by using the response surface method and the MOGA genetic algorithm.

\section{RESULTS AND ANALYSIS}

Numerical calculations were carried out, using the mesh finite element method, assuming three lengths of a straightened mini robot resulting from its working space. The first length of the mini robot is equal to $175.52 \mathrm{~mm}$ and six shapes of deformation are illustrated in the Figures 6-11. For the $\lambda$ equals to 5.24 the mini robot is deformed in plane XZ. For the second eigenvalue, the mini robot is deformed in orthogonal direction to YZ plane. Third shape is similar to the deformation in first direction and fourth is similar to the second shape of deformation. The fifth shape shows the deformation of the mini robot in the XZ plane and the sixth in the $\mathrm{YZ}$ plane.

In Figures 12-15, there are shown deformations of $270.52 \mathrm{~mm}$ effector long. The qualitatively obtained deformations of shapes are similar to those obtained for the suitable mini robot forms with a length of $175.52 \mathrm{~mm}$. In Figures 16-19, there are shown deformations of $350.52 \mathrm{~mm}$ effector long for the next load factors.

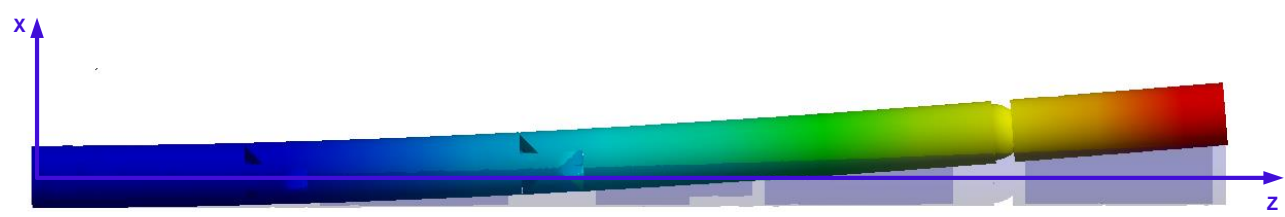

Figure 6. First shape of deformation for $\lambda$ equals 5.24

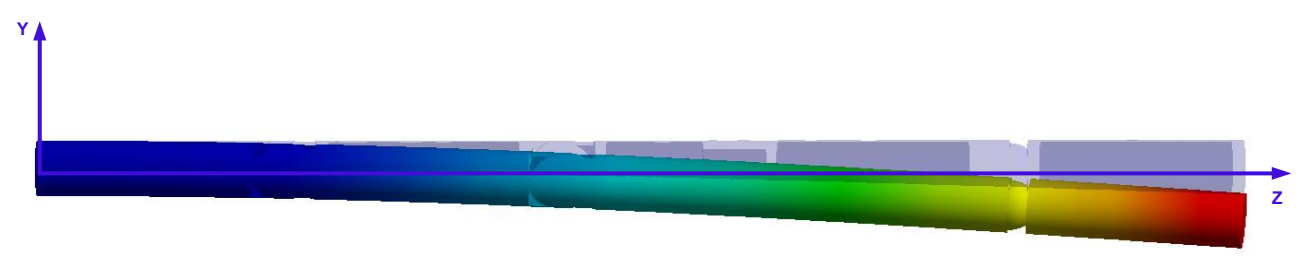

Figure 7. Second shape of deformation for $\lambda$ equals 5.58 


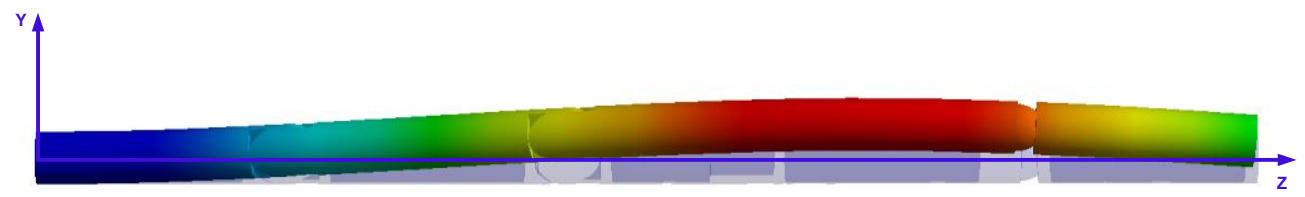

Figure 8 . Third shape of deformation for $\lambda$ equals 47.37

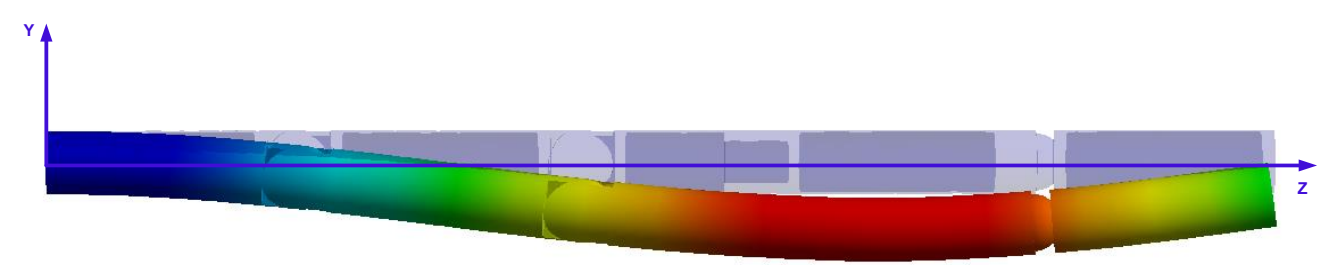

Figure 9. Fourth shape of deformation for $\lambda$ equals 47.74

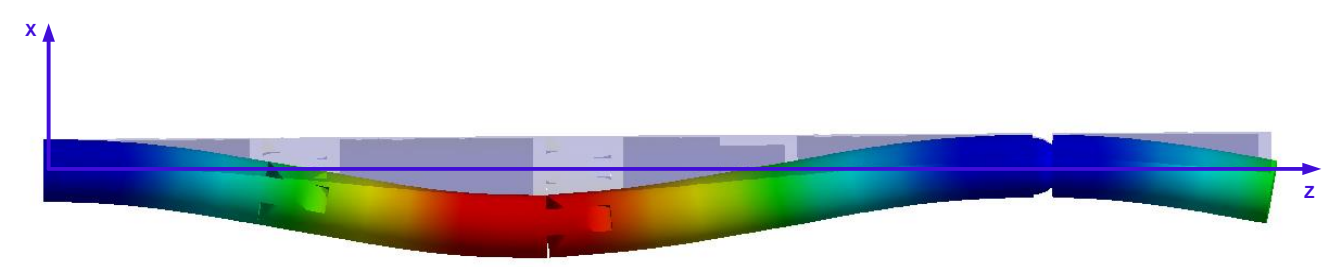

Figure 10. Fifth shape of deformation for $\lambda$ equals 120.47

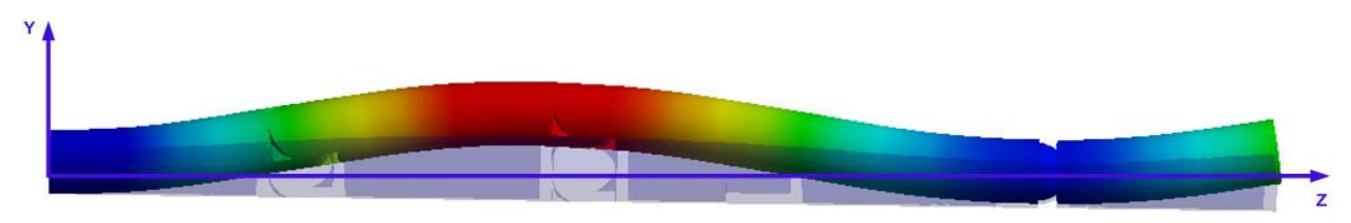

Figure 11. Sixth shape of deformation for $\lambda$ equals 125.98

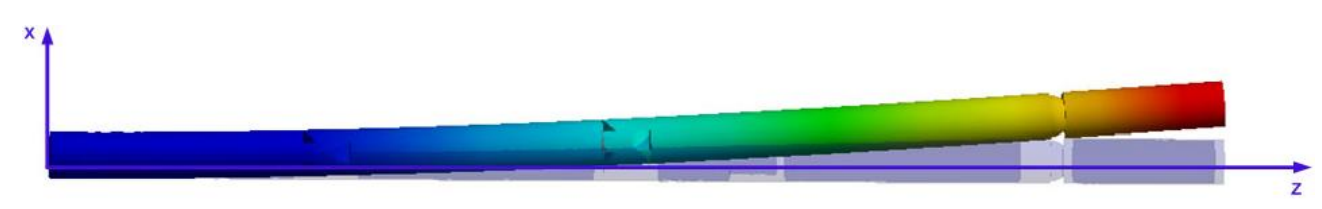

Figure 12. First shape of deformation for $\lambda$ equals 2.87

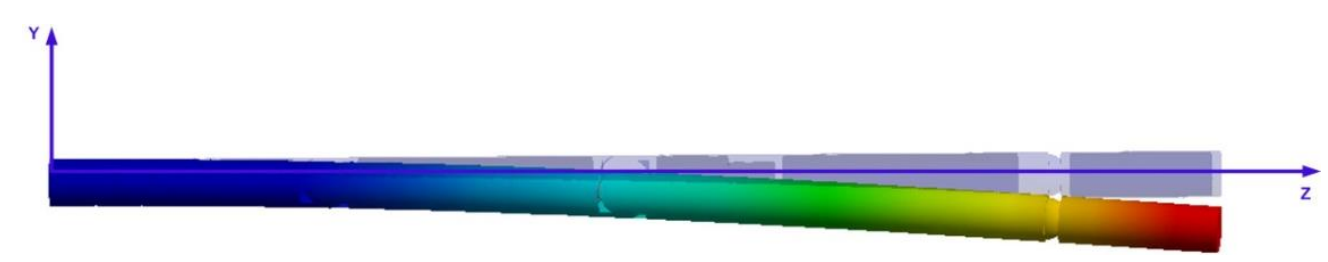

Figure 13. Second shape of deformation for $\lambda$ equals 2.97

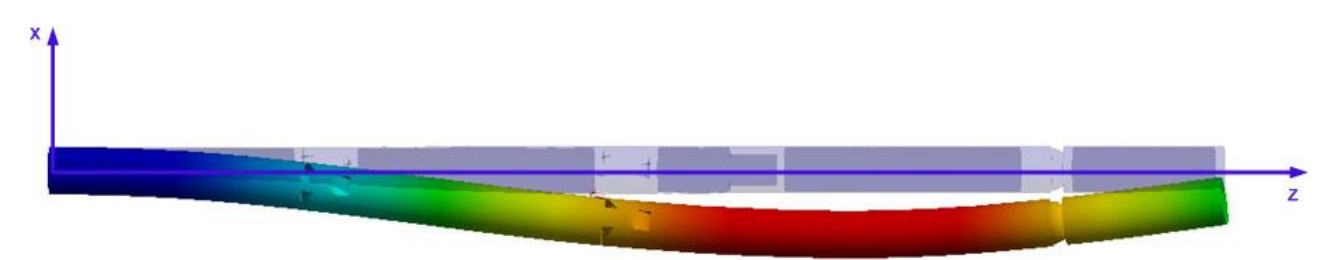

Figure 14. Third shape of deformation for $\lambda$ equals 26.17 


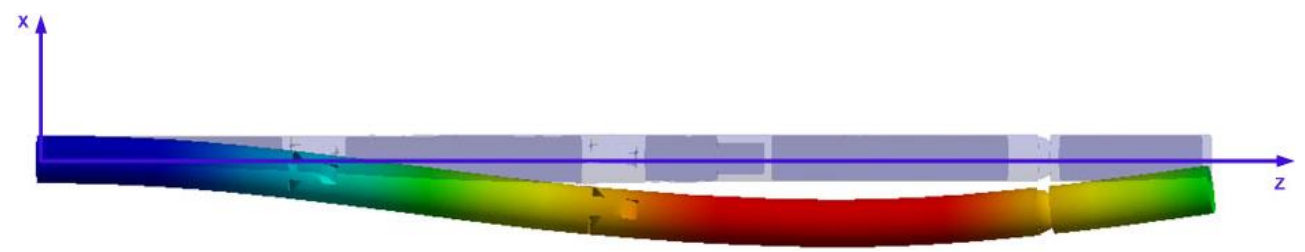

Figure 15. Fourth shape of deformation for $\lambda$ equals 26.38

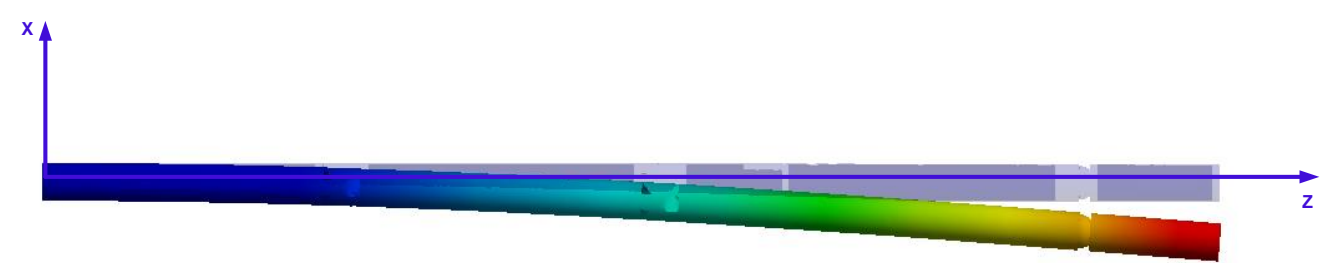

Figure 16. First shape of deformation for $\lambda$ equals 1.83

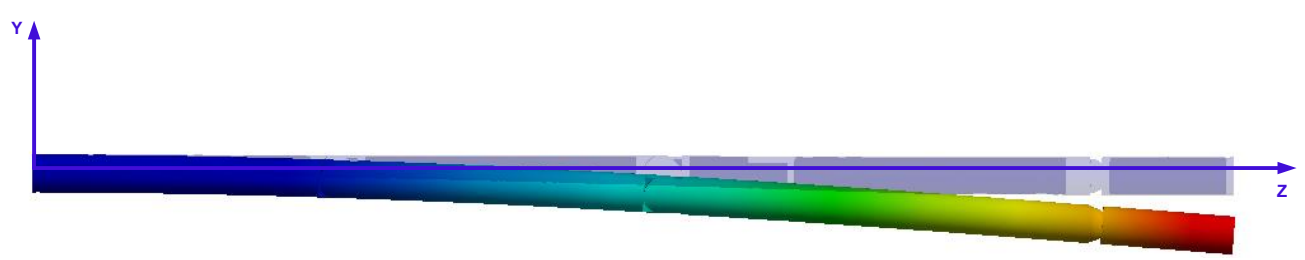

Figure 17. Second shape of deformation for $\lambda$ equals 1.88

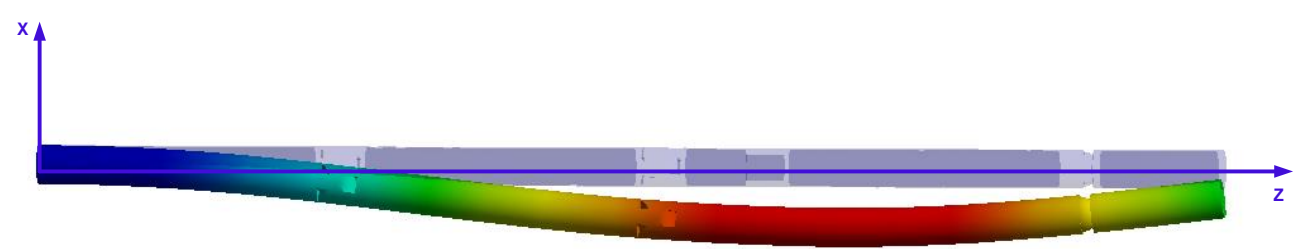

Figure 18. Third shape of deformation for $\lambda$ equals 16.64

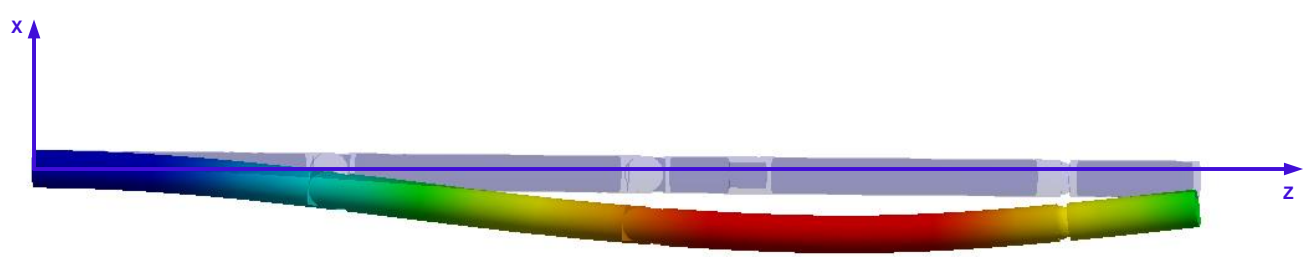

Figure 19. Fourth shape of deformation for $\lambda$ equals 16.78

On the basis of results obtained in three experiments for three lengths of the effector, it can be found that the load factor increases its value when the length increases, which is coherent with the prediction. The obtained results are important from superior importance point of view, which is the safety criterion of using a medical robot. The lowest values of the load factor were obtained for the longest effector with a length of $350.52 \mathrm{~mm}$. The $\lambda$ equals 1.83 .

\subsection{Optimization results}

The response surface for the criterion of mass is shown in Figure 20. This figure makes it possible to determine the value of the mini robot's mass, depending from the dimensions of $d_{1}$ and $d_{2}$, from which depend the thickness of robot's wall. The metamodel (response surface) served also as a continuous multidimensional function (obtained on the basis of numerical experiments of FEM) from which the MOGA genetic algorithm found the optimal solution. The solution was generated on the basis of Pareto fronts. The Pareto fronts for the criterion of mass are shown in Figure 21. Convergence to the result was 
obtained after the 23 iterations. On the basis of Pareto's fronts optimal shape of mini robot was obtained for two criterions.

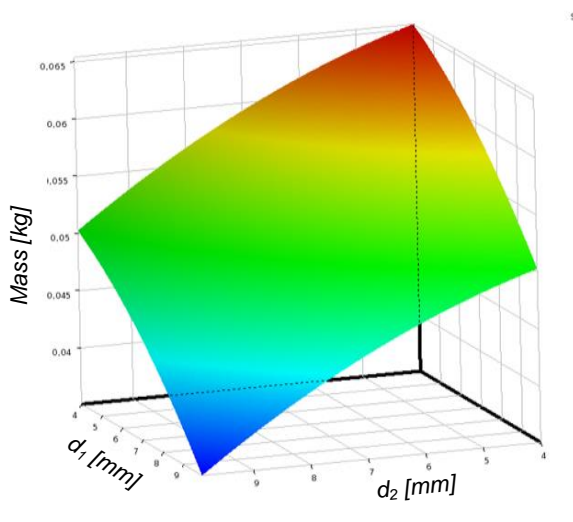

Figure 20. Response surface for mass

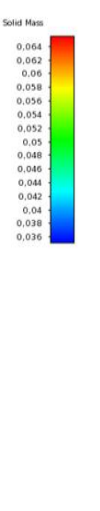

Figure 21. The Pareto fronts for the criterion of mass

\section{DISCUSSION}

The medical robot is currently one of the most significant devices for operating the human body. Medical robots with a serial chain are innovative structures, for example: in contrast to the American structure of the da Vinci or the Polish prototype of Robin Heart. It is expected that in the near future, many modern medical robots will be created, which will operate in oncology, heart surgery, abdominal surgery, etc. Restrictive safety criteria require many structural analyzes. These can include: analysis of natural frequency, fatigue, transient states, to protect it against the possibility of a robot's failure during the surgery on human tissue. An effective method of examining a medical robot system is a numerical method based on the finite element method. While using a mesh model with a small finite element, exact results can be obtained. This method can be checked, if a medical robot with a serial chain will be prone to buckling during the contact with the tissue (e.g. hard bone tissue). FEM has been successfully used in medical robot analyzes many times. The discussed issues are calculated relatively quickly with the usage of modern computers currently available in numerical labs. The optimization calculations take the most time. The MOGA genetic algorithm makes it possible to find the optimum of a global multi-criterion function.

\section{CONCLUSIONS}

The work presents an innovative model of a medical robot with an open kinematic chain. Due to the criterion with superior importance, which is safety, numerical examinations of the robot for the buckling with the use of the finite element method was performed in order to check if it can occur during the robot's work, for example during the servicing of an artificial organ. For discretization of the geometric model, tetrahedral elements with ten nodes were used. Based on numerical examinations, it was found that the buckling phenomenon will not occur in the structure of a medical robot with a serial chain.

The numerical Multi-criteria optimization model was also solved with the usage of MOGA genetic algorithm and the minimum mass of the effector and the maximum value of the buckling coefficient were obtained. The plane response method was used in the optimization researches. Whereas the optimal solution was generated on the basis of designated Pareto fronts. A mechanically proper calculation was obtained due to the accepted criteria and limitations.

\section{REFERENCES}

[1] G. Ilewicz, "Natural frequencies and multi - objective optimization of the model of medical robot with serial kinematical chain," Advances in Intelligent Systems and Computing, vol. 519, pp. 371-379, 2017.

[2] G. Ilewicz, A. Harlecki, "Full Dynamics and Optimization of a Controllable Minimally Invasive Robot for a Soft Tissue Surgery and Servicing Artificial Organs," Lecture notes in Network and Systems, vol. 53, pp. 204-211, 2019.

[3] G. Ilewicz, "Strength of materials," Center for Microelectronics and Nanotechnology (in Polish), Rzeszów, 2013.

[4] H. Wang, A. Yamamoto, "Analyses and Solutions for the Buckling of Thin and Flexible Electrostatic Inchworm Climbing Robots," IEEE Transactions on Robotics, vol. 33, no. 4, 2011. 
[5] M. Bourezane, "Buckling finite element analysis of beams and frame," Proceedings of the World Congress on Engineering, vol. I, London, U.K., 2012.

[6] R. Konietschke, T. Ortmaier, H. Weiss, R. Engelke, G. Hirzinger, "Optimal Design of a Medical Robot for Minimally Invasive Surgery, 2," Jahrestagung der Deutschen Gesellschaft für Computer-und Roboterassistierte Chirurgie (CURAC), Nürnberg, Nov. 4-7, 2003.

[7] M. Miroir, Y. Nguyen, J. Szewczyk, S. Mazalaigue, E. Ferrary, O. Sterkers, A. Grayeli, "RobOtol, From Design to Evaluation of a Robot for Middle Ear Surgery," in The 2010 IEEE/RSJ International Conference on Intelligent Robots and Systems, 2010.

[8] M. Miroir, J. Szewczyk, Y. Nguyen, S. Mazalaigue, O. Sterkers, "Design of a robotic system for minimally invasive surgery of the middle ear," in 2nd IEEE RAS \& EMBS International Conference on Biomedical Robotics and Biomechatronics, 2008.

[9] C. Drouin, A. Pourghodrat, S. Miossec, G. Poisson, C. Nelson, "Dimensional optimization of A TWO-ARM robot for single-site surgery operations," in ASME 2013 International Design Engineering Technical Conferences and Computers and Information in Engineering Conference, Portland, United States, Aug 2013.

[10] L. Bostic, S. Lanczos, "Eigensolution method for high-performance computers," National Aeronautics and Space Administration Memorandum, Langley Research Center, 1991.

[11] O. Zienkiewicz, R. Taylor, J. Zhu, Finite element method: Its basis and fundamentals, Elsevier, 2013

\section{BIOGRAPHIES OF AUTHORS}

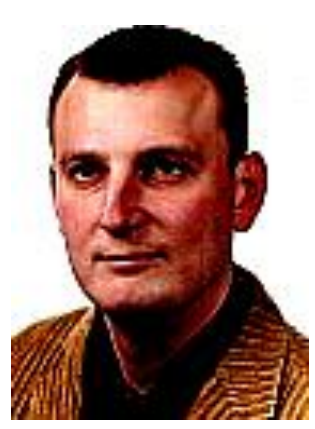

Grzegorz ILEWICZ was born in Katowice, Poland on December, 1980. Up to this moment, he has been an employee of four universities in the fields of mechatronics, biomechatronics, mechanics and automation. He is a member of Polish Acoustical Society. He held a didactic seminar at CERN and Aachen University. Until then, He has been the general chairman of six technical conferences. He has been a member of several dozen conference committees around the world. He is also in the editorial boards of technical and medical journals.

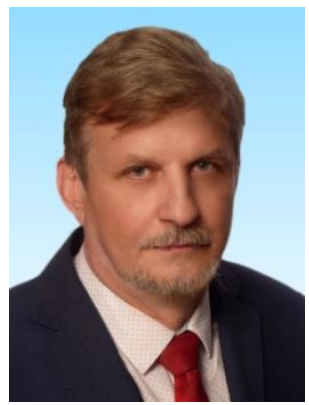

Andrzej HARLECKI was born in Bielsko-Biała, Poland on August 6, 1955. He received the M.Sc. and Ph.D. degrees in thefield of Machine Design and Maintenance from Technical University of Lodz (Branch in Bielsko-Biala) in 1980 and 1989, respectively. He received the D.Sc. degreein the field of Machine Design and Maintenance from University of Bielsko-Biala in 2003. Since 2004 he is employed by the University of Bielsko-Biala as an associate professor. $\mathrm{He}$ is an authoror co-author of 130 publications in scientific journals and conference proceedings. He is now the Head of independent Section of Mechanics associated with the Faculty of Mechanical Engineering and Computer Sciences of the University of Bielsko-Biala. His papers and research covers various disciplines of mechanics (in particular modelling of friction in dynamics of mechanical systems), tribology, mechatronics and computer sciences.He was supervisor of three doctoral theses. He directed over a dozen industry research projects. 